\title{
Open Conservation: tecniche di rappresentazione a supporto dell'iter conservativo
}

\author{
Francesca Gasparetto \\ Laura Baratin
}

\section{Abstract}

L'ambito dei beni culturali è stato recentemente interessato da un processo di digitalizzazione che sta evolvendo e sta radicalmente modificando il significato di fruizione e valorizzazione. Nonostante le tecnologie e le sperimentazioni messe a servizio del settore siano in costante crescita, è ancora difficile definire il loro ruolo in un'ottica di 'conservazione in digitale'.

II contributo propone un nuovo approccio alla progettazione delle pratiche conservative a partire dall'impiego dei dati digitali che un cantiere di restauro può produrre e alla loro diversa rappresentazione.

Consapevoli che i processi tecnici di digitalizzazione non sono sufficienti all'ottenimento di una corretta conservazione, è necessario approfondire una ricerca in grado di proporre un approccio metodologico, organizzativo e procedurale per l'evoluzione della nuova conservazione. Il lavoro propone un flusso organizzato in tre livelli narrativi. Ognuno di questi impiega approcci specifici, al fine di rendere accessibile la documentazione digitale prodotta nell'ambito di cantieri di restauro aperti.

L'obiettivo generale è quello di definire un nuovo indirizzo teorico, in grado di includere nel processo conservativo l'impiego delle recenti tecnologie in modo consapevole e gettare le basi per lo sviluppo dei lineamenti di una teoria inter- e multidisciplinare che guidi il futuro delle pratiche conservative secondo una logica digitale.

\section{Parole chiave}

conservazione, accessibilità, partecipazione, metodologia digitale, livelli narrativi.
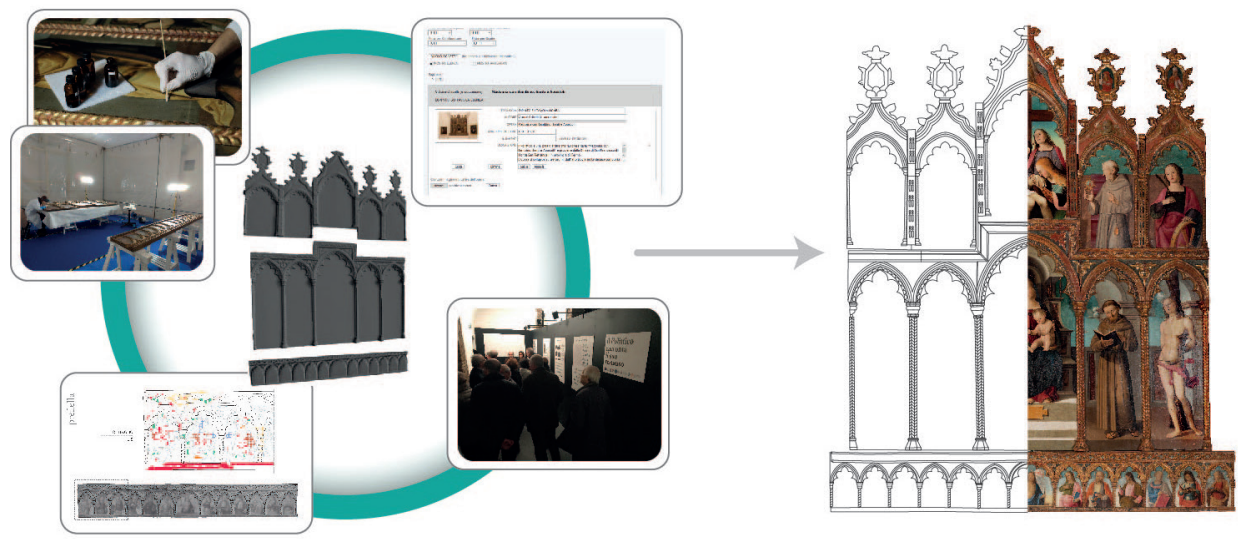


\section{Introduzione}

Nell'ambito dei Beni Culturali, in particolar modo per quanto riguarda la fruizione e la valorizzazione, è universalmente riconosciuto il ruolo della documentazione e della rappresentazione digitale per scopi di tipo divulgativo. Le applicazioni e la digitalizzazione di numerose collezioni museali hanno negli ultimi anni sensibilizzato il pubblico all'utilizzo di tecnologie sempre più avanzate. Diverso è lo stato dell'arte se parliamo di conservazione e restauro, ambito che rimane ancora oggi argomento tecnico specifico a cui si interessano principalmente esperti del settore.

L'obiettivo del lavoro presentato è di dimostrare come, per quanto oggi si parli di applicazioni digitali per i Beni Culturali, sia ancora necessario approfondire e definire l'ambito della conservazione in digitale del patrimonio e del suo valore. Ciò può avvenire attraverso il riconoscimento del ruolo del restauratore/conservatore - oggi formato riguardo le principali tecnologie e tecniche di documentazione - all'interno della filiera produttiva. II processo conservativo in digitale, infatti, vede l'utilizzo degli strumenti contemporanei come un'occasione per innovare teoria, pratica e obiettivi generali, al fine di rendere il Patrimonio sempre più accessibile.
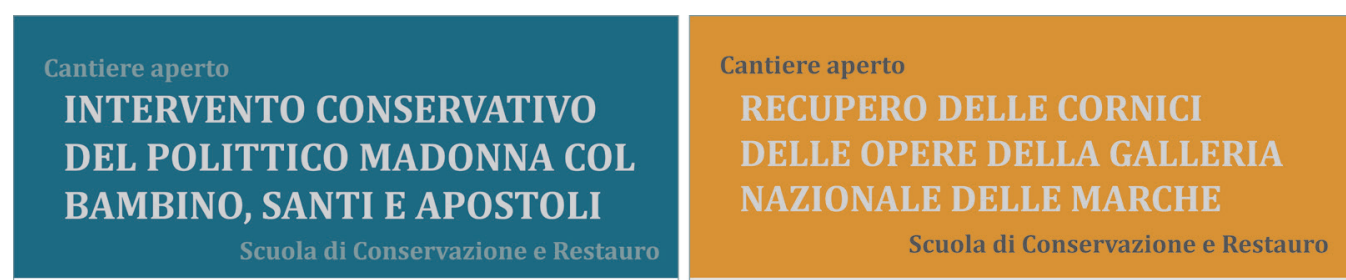

CONVENZIONE TRA ANCI MARCHE | PIO SODALIZIO DEI PICENI | UNIVERSITA' DI URBINO 4 ottobre 2018 delibera n. $140 / 2018$ Convenzione per indagini al fine del
restauro di opere d'arte conservate ne Comuni marchigiani lesionate da

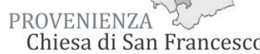
Monte San Pietrangeli (FM)
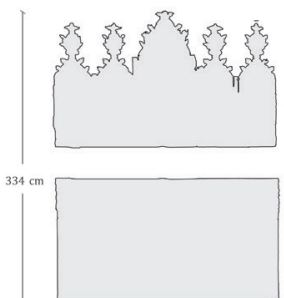

part
assemblate

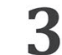

personaggi raffigurati raffigurati

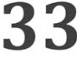

di lavoro 5

ANALISI STORICO-ARTISTICHE 1 RILIEVI GEOMETRICI 2D E 3D 2 INDAGINI DIAGNOSTICHE $\mathbf{3}$ PROGETTO E INTERVENTO 4 COMUNICAZIONE E FRUIZIONE 5

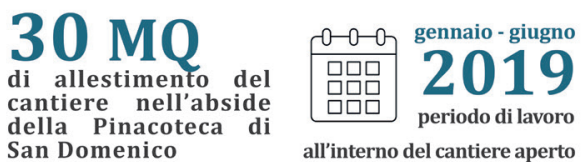
San Domenico all'interno del cantiere aperto

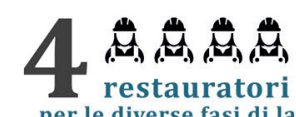

per le diverse fasi di lavoro: dalla movimentazione all'intervento

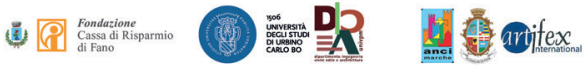

Schedatura digitale e Mappatura dello stato conservativo delle cornici o parti di cornici conservate nei depositi di Palazzo Ducale

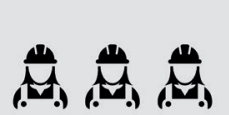

3 restauratori

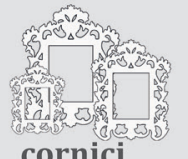

cornici o parti di cornic
La schedatura prevede l'acquisizione di informazioni relative a:

$>$ caratteristiche storico-artistiche

$>$ rilievi dimensionali

$>$ materiali costitutivi

$>$ stato conservativo

questo porta alla redazione di schede conservative dedicate utilizzando i sistemi digitali di documentazione dell'Università di Urbino.

Larchivio digitale fornisce alla Direzione della Galleria Nazionale delle Marche uno strumento per la programmazione degli interventi, al fine del riutilizzo delle cornici nell'allestimento museale $\mathrm{e}$ una ricerca della loro collocazione originale,
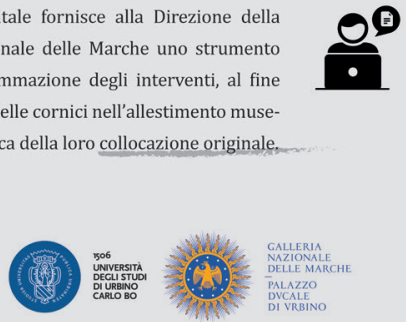


\section{La conservazione in digitale e il processo europeo per il suo riconoscimento}

Negli ultimi anni, in particolare a seguito del 2018 [I], si è concretizzato sempre di più l'impegno delle istituzioni europee nel riconoscere un preciso spazio culturale alla produzione digitale proveniente da studi e indagini sul Patrimonio. In particolare, la Commissione Europea si è espressa attraverso l'approvazione di carte e documenti dedicati al tema dell'accessibilità e della valorizzazione attraverso il digitale. Un esempio su tutti è la Dichiarazione di cooperazione riguardo il progresso della digitalizzazione firmata nel 2019 da 27 Paesi membri [2], nella quale i firmatari si impegnano a sostenere politiche culturali mirate alla digitalizzazione dei monumenti e dei siti del patrimonio e all'impiego di risorse digitali per promuovere il coinvolgimento dei cittadini in attività innovative di salvaguardia e protezione.

L'interesse al tema è dimostrato anche dai finanziamenti a supporto di importanti progetti di ricerca. II riferimento più recente è a $4 \mathrm{CH}$ project [3], un progetto finanziato nell'ambito di H2020 cominciato nel gennaio 202I il cui scopo principale è la costruzione di un Competence Centre per la conservazione del Cultural Heritage, attraverso applicazioni totalmente digitali.

I ricercatori impegnati nel campo della documentazione e della digitalizzazione ormai da qualche anno stanno dichiarando la necessità di definire nuovi ruoli di competenza per poter costruire al meglio il nuovo settore del digitale [Munster 2017]. Servono infatti etica operativa [Santana Quintero 2019], individuazione dei corretti canali di trasmissione e soprattutto standard a cui fare riferimento [loannides et al. 2005; Gasparetto, Baratin 2020] al fine di operare tutti nella stessa direzione.

Se innovare significa occuparsi e introdurre l'utilizzo di strumenti contemporanei per migliorare la pratica di salvaguardia del Patrimonio, è altrettanto importante individuare quali cambiamenti teorici può comportare l'impiego di nuove tecnologie. La Conservazione 3.0 proposta da Gustafsson [Gustafsson 2019] tiene in considerazione il cambiamento di scenario globale e l'evoluzione della cultura sociale, per proporre una conservazione più attiva degli oggetti culturali. Gli attori di questo nuovo processo lavorano su più livelli, perché la conservazione non risponde alle sole esigenze dei beni, ma anche a quelle dei cittadini, entrando così in una zona di scambio tra esigenze pratiche e necessità teoriche da rispettare. In questo senso, chi parla di conservazione oggi deve mettere in relazione i nuovi strumenti con le necessità di una società dell'informazione sempre più evoluta e più responsive, progettando un approccio partecipativo che conserva edifici e opere d'arte così come preserva le relazioni culturali tra oggetti e cittadini.

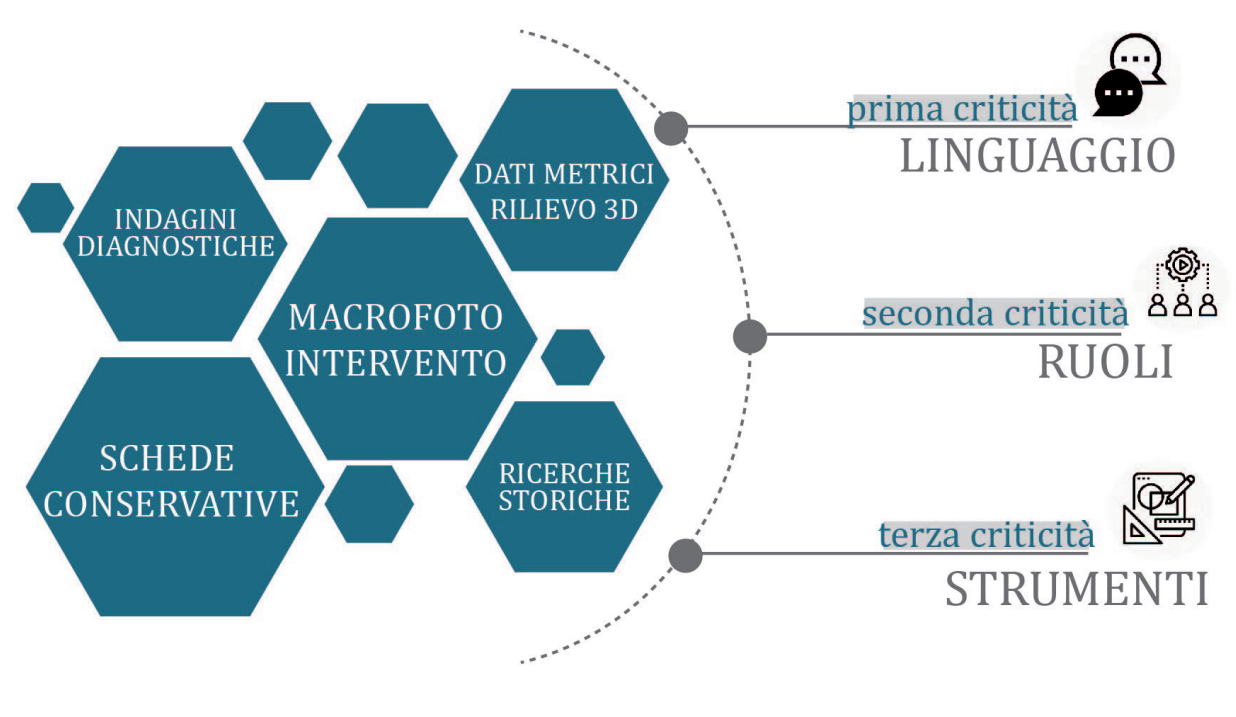




\section{Una proposta metodologica}

La Scuola di Conservazione e Restauro dell'Università di Urbino sta sviluppando, da alcuni anni, la tematica del ruolo del "restauratore digitale" nello scenario contemporaneo, sia nell'ambito della ricerca sia all'interno della didattica. In particolare, l'offerta formativa presenta due insegnamenti dedicati alla documentazione e alle tecniche di rappresentazione digitali e - all'interno delle attività pratico laboratoriali - ulteriori approfondimenti riguardo le elaborazioni tridimensionali e il loro uso nell'ambito della mappatura dei fenomeni di degrado. II piano di studi, infatti, prevede il trasferimento di nozioni di tipo tecnico-documentativo al fine di fornire agli studenti i lineamenti della scienza della rappresentazione e della comunicazione applicati agli interventi di restauro. Alla stessa maniera le attività di terza missione sono spesso rivolte al coinvolgimento della cittadinanza e dei visitatori nelle mostre organizzate, a partire da ricerche progettate e condotte intorno alla tematica della conservazione e del restauro in digitale.

II caso studio più emblematico condotto nell'ultimo periodo è l'allestimento di due cantieri aperti dedicati rispettivamente al restauro del Polittico di Monte San Pietrangeli presso la Pinacoteca di San Domenico a Fano e all'analisi e alla documentazione delle cornici conservate all'interno dei depositi di Palazzo Ducale a Urbino (fig. I).

L'obiettivo di entrambe le proposte era di costruire uno scenario di conservazione proattiva, che passa dalla documentazione del lavoro svolto in cantiere per poi ottenere informazioni divulgative e che stimolano la partecipazione di visitatori e cittadini.

Una prima parte di sperimentazione si è concentrata sull'impostazione di un workflow documentativo ottimale, in grado di mettere in relazione le tecnologie digitali impiegate e sviluppare un modello standard orientato alla raccolta dinamica dei dati [Baratin, Gasparetto 2019; Baratin, Gasparetto 2020].

La seconda fase si è indirizzata alla ricerca di un metodo comunicativo da applicare agli interventi, durante lo svolgimento e dopo la chiusura dei cantieri.

Questo tipo di ricerca è stato condotto in diversi contesti. Per le cornici si è trattato di una breve esperienza, con un cantiere allestito all'interno degli spazi dei sotterranei di Palazzo Ducale a Urbino, interrotta dalla situazione emergenziale del Covid- 19 che non ha permesso di sviluppare ulteriormente le strategie comunicative avviate. Per il Polittico, invece, le occasioni sono state molteplici: dal cantiere aperto di Fano allestito durante il 2019, alle mostre in cui l'opera è stata esposta ad Ascoli Piceno, a Roma e a Senigallia tra il 2020 e il 202 I, fino alla fase di progettazione che sta interessando l'ambiente in cui verrà ricollocata l'opera a Monte San Pietrangeli verso la metà del 202 I. Gli spazi e le necessità, che le situazioni diverse richiedevano, hanno portato all'evolversi di opportunità di sviluppo e integrazione di applicazioni ibride tra digitale e analogico. A partire dall'analisi delle criticità (fig. 2) che lo scenario proponeva, si è proceduto alla definizione di un modello di comunicazione del processo conservativo. La prima criticità riguardava la mancanza di un linguaggio concordato e riconosciuto da applicare per comunicare i dati raccolti. Infatti, normalmente durante un cantiere si elaborano molti dati tecnici e scientifici, sistematizzati all'interno di un sistema documentativo appositamente progettato, ma non in grado di tradurre i dati in informazioni. In questo caso, gli strumenti digitali - pur fornendo un supporto fondamentale per la qualità dell'analisi e della gestione dati - non sono in grado di elaborare questo tipo di traduzione. Si è trattato quindi di sviluppare un sistema di open data, che desse la possibilità ai visitatori di indagare ed entrare all'interno del sistema documentativo, secondo specifiche autorizzazioni.

La seconda criticità ha interessato la definizione dei ruoli. Infatti, se la normativa italiana in fatto di Patrimonio culturale [4] indica chiaramente chi deve occuparsi di restauro, di sicurezza, di valorizzazione e di tutela, non è chiaro e non sono mai state palesemente definite le competenze e professionalità di chi si occupa di comunicazione legata all'incremento dell'accessibilità ai beni culturali e delle loro vicende conservative. L'ambiguità dei ruoli porta a un conseguente malinteso, soprattutto sul piano tecnologico, non permettendo ai restauratori, responsabili in prima persona dell'esito estetico e comunicativo degli interventi, di sentirsi direttamente coinvolti nello sviluppo dei contenuti digitali sia durante che dopo il processo conservativo. 
Infine, la terza criticità riscontrata riguarda l'approccio ai dati e la scelta degli strumenti tecnici e delle tecnologie necessarie per la loro acquisizione e successivamente per la divulgazione. Riconoscendo, infatti, la varietà degli strumenti a disposizione, mancano oggi linee guida e protocolli operativi condivisi che validino in modo ufficiale l'impiego di tecnologie specifiche per scopi altrettanto peculiari. La Carta di Londra già più di dieci anni fa sottolineava l'importanza di definire degli standard di visualizzazione in digitale, necessari per potersi muovere in un contesto scientificamente approvato. Allo stesso modo, sarebbe necessario oggi far riferimento a principi generali che regolano l'uso di tecnologie digitali, nel contesto della protezione e della salvaguardia del patrimonio.

Il lavoro svolto per il Polittico, e inizialmente per le cornici dei depositi di Palazzo Ducale, ha permesso di strutturare un modello, ancora in corso di sperimentazione, che a partire dell'analisi dei materiali raccolti durante il restauro e dalla destrutturazione degli output ottenuti per la comunicazione (visite guidate ai cantieri, mostre itineranti dell'opera restaurata, ecc.) permettesse di disegnare un piano di attività per condividere in più direzioni i contenuti del progetto.
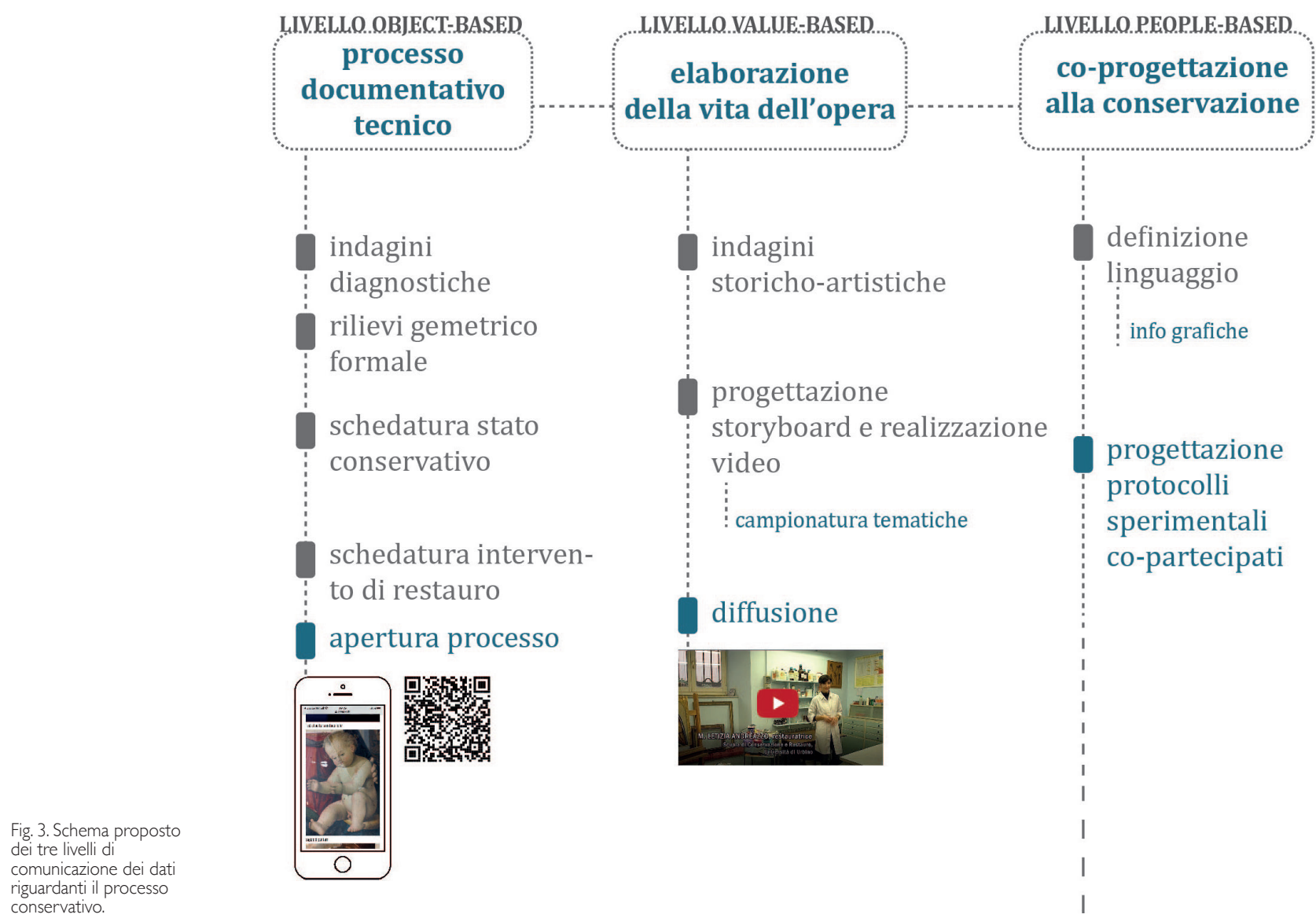

Una proposta di tre livelli narrativi per una conservazione aperta del patrimonio restaurato

La proposta, sperimentata sul Polittico di Monte San Pietrangeli, è stata organizzata su tre livelli narrativi: uno di tipo tecnico-scientifico, uno di tipo storico-artistico e infine uno di tipo socioculturale e identitario, ai quali sono corrisposti strumenti diversi di rappresentazione delle informazioni raccolte (fig. 3), organizzando il flusso documentativo secondo output progettati in maniera specifica per ogni livello. 
II primo livello ha messo a sistema tutte le informazioni più specialistiche legate alla forma, alle dimensioni, ai materiali e agli interventi di restauro; si tratta di schede descrittive dello stato conservativo, fotografie di dettaglio dei degradi, ecc. fino agli appunti tecnici raccolti durante le operazioni di cantiere. Sono stati definiti i responsabili di questa raccolta, individuati tra i restauratori, i diagnosti e gli esperti di settore. Il livello narrativo è stato nominato 'object-based', poiché la tipologia di rappresentazione del dato raccolto è strettamente legata all'opera d'arte e alla conservazione della sua fisicità.

II secondo livello ha definito il 'viaggio' del Polittico basato sulla raccolta dei dati storici che collocavano l'opera da un punto di vista artistico e ne mostravano la sua complessità. II livello chiamato value-based vuole sottolineare l'importanza della narrazione storico-artistica e focalizzare di conseguenza il valore dell'oggetto. I due livelli narrativi descritti vengono riconosciuti come momenti passivi, durante i quali il visitatore assorbe informazioni e le rielabora individualmente.

Infine, il livello socioculturale e identitario, chiamato people-based, definisce un ecosistema integrato compartecipato, all'interno del quale non c'è un ruolo definitivo, ma l'attività della comunità di cittadini si integra con quella di esperti, collaborando così all'evoluzione del $\mathrm{Pa}$ trimonio. In questo step è necessaria l'interazione tra le diverse parti, intesa come motore produttivo, creando quindi un processo attivo.

È interessante analizzare i contesti in cui i tre livelli si possono sviluppare: nel caso del cantiere aperto, i livelli utilizzati per la comunicazione sono i primi due. Qui la rappresentazione ha impiegato strumenti digitali per veicolare gli step del lavoro e renderlo più comprensibile ai non esperti, attraverso la possibilità di inquadrare un $\mathrm{QR}$ code che collega le schede ed eventuali macrofoto dell'intervento. Nonostante il dato venga comunicato passivamente, è stato però necessario individuare uno strumento che permettesse un aggiornamento continuo dell'evoluzione del lavoro.

Diverso è il progetto per la ricollocazione del dipinto, che prevede la compresenza dei tre livelli narrativi. In questo caso, anche l'output finale per ogni livello è diverso. Infatti, i primi due raccontano un lavoro concluso e per questo si propone di rappresentare le informazioni attraverso strumenti definiti "fissi", come pannelli esplicativi o proiezioni video.

Al livello object-based è stata associata una rappresentazione che non richiede interazione col pubblico, ma piuttosto una sistematizzazione del dato elaborato da tecnici durante

Fig. 4. Esempio di pannello esplicativo da esposizione che riporta informazioni riguardo le tecniche esecutive e lo stato di conservazione della parte alta del Politico Questo tipo di pannello Qur tipo di pannello sarà esposto all interno dello spazio polifunzionale e riguarda il livello objectbased.

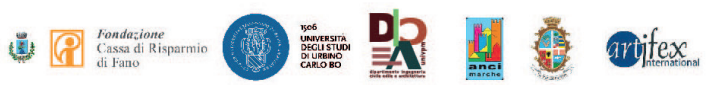

INTERVENTO CONSERVATIVO DEL POLITTICO MADONNA COL BAMBINO, SANTI E APOSTOLI
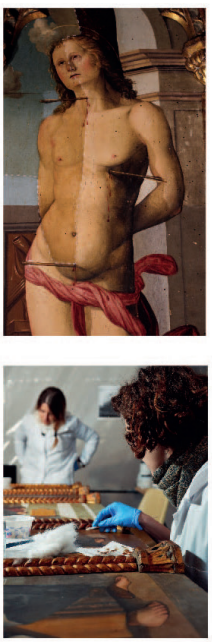
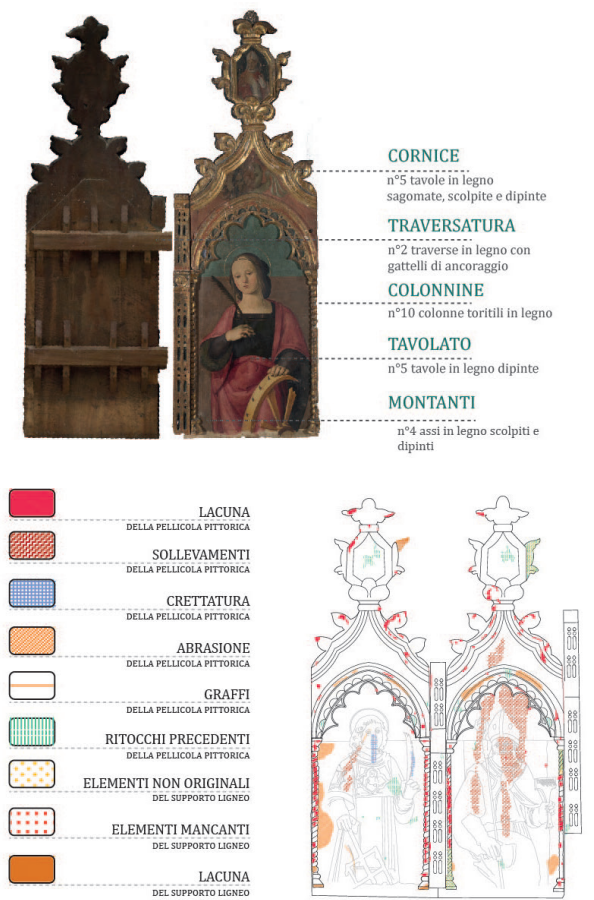
le analisi e l'intervento. A partire da dati digitali di rilievo, sono state realizzate le mappature bidimensionali (2D) con metodi di rappresentazione tecnica consolidati in ambito architettonico in grado di riassumere i risultati delle indagini, sfruttando la potenza comunicativa della semplificazione grafica e di lettura semantica del colore (fig. 4).

Per il livello value-based si è scelto lo strumento video, più consono per trasmettere l'idea di evoluzione e costruzione della conoscenza, impiegando immagini in movimento, a supporto della descrizione di un dato in continua evoluzione, soggetto alla storia e al tempo. Attraverso un lavoro di campionatura delle tematiche, sono stati realizzati brevi video di facile interpretazione, che coinvolgono gli attori e i diversi processi di restauro e progetti di valorizzazione [5].

Grazie all'introduzione alla tematica conservativa offerta dalla fruizione dei due livelli narrativi descritti, è possibile predisporre la progettazione di protocolli co-partecipati per la conservazione e la fruizione. II livello narrativo introdotto in questa fase è quello people-based e il lavoro si organizza in due momenti: uno progettuale chiuso e l'altro partecipato aperto. La prima fase progettuale vede la definizione di un linguaggio grafico da far utilizzare in maniera autogestita nella fase partecipata: sono state selezionate alcune infografiche per indicare le macrotematiche, già definite nel livello precedente, e sono indicati alcuni colori specifici, a loro volta già utilizzati per la rappresentazione del degrado del livello object-based. Lo spazio espositivo in cui l'opera verrà ricollocata è un ambiente polifunzionale, che verrà impiegato dalla comunità del paese di Monte San Pietrangeli a vari scopi. L'intento dell'amministrazione è quello di poter far vivere il Polittico dopo l'importante restauro, non solo attraverso la tradizionale fruizione museale, ma anche attraverso una partecipazione attiva. Infatti, dal momento che l'opera rientrerà dopo le diverse mostre itineranti circa a metà 202I, sono state programmate attività specifiche di valorizzazione e di fruizione dell'opera stessa in un'ottica di riappropriazione del patrimonio danneggiato dal terremoto del 2016. In questo senso, gli strumenti grafici progettati verranno impiegati a vari livelli per continuare a scrivere la storia del Polittico. Per quanto riguarda i metodi rappresentativi dei risultati che si otterranno, questi non sono ancora stati definiti poiché il processo partecipativo porterà alla scelta più idonea seguendo il metodo proposto.

\section{Conclusioni}

I metodi di rappresentazione impiegati e quelli proposti sono rivolti alla creazione di un processo conservativo e non di un prodotto per la conservazione; ciò permette di adattarsi ai continui cambiamenti, alle necessità del Patrimonio e della comunità che lo custodisce. II lavoro proposto presenta una possibile traduzione di un processo conservativo in un'ottica di contemporaneità. Esprimersi attraverso livelli comunicativi calibrati alle fasi di intervento permette - in ultima battuta - di progettare un'apertura del processo conservativo, rendendolo accessibile nelle sue fasi più tecniche, accorciando così le distanze tra esperti e comunità di appartenenza del Patrimonio. Le competenze e gli strumenti delle discipline della rappresentazione, unitamente al coordinamento delle diverse tecnologie a disposizione per tutte le fasi di acquisizione, elaborazione e gestione dei dati, ha portato alla definizione di un modello informativo people-centred.

L'approccio tipicamente digitale - che preferisce la costruzione di un network partecipato piuttosto che un sistema informativo unidirezionale - permette di mettere a sistema le molte informazioni a disposizione e rendere veramente attivi i dati raccolti durante un intervento di restauro e più in generale lungo un percorso conservativo.

\section{Note}

[I] Anno Europeo per il Patrimonio Culturale: <https://europa.eu/cultural-heritage> (consultato il I0 gennaio 202 I).

[2] <https://ec.europa.eu/digital-single-market/en/news/eu-member-states-sign-cooperate-digitising-cultural-heritage(consultato il 10 gennaio 2021 ). 
[3] <https://www.4ch-project.eu> (consultato il I0 gennaio 202I).

[4] Si fa riferimento al Codice dei Beni Culturali e del Paesaggio (D.Lgs 42/2004, art. 30).

[5] Non ci sono immagini dell'allestimento del video all'interno della stanza poiché l'inaugurazione dello spazio polifunzionale è stato rinviato a causa dell'emergenza Covid- 19.

\section{Riferimenti bibliografici}

Baratin L., Gasparetto F. (2019). Contemporary restoration as an inclusive process. The new digital documentation challenges. Disegnarecon, G. Amoruso, T. Jelenski (a cura di). Experiential Design for Heritage and Environmental Representation, vol. I2, n. 23, pp. 3.1-3.13.

Baratin L., Gasparetto F. (2020). Digital Cultural Heritage: some notes from conservative experience. In C. Gambardella (ed.). World Heritage and Contamination. Abstracts of the XVIII International Forum 'World Heritage and Contamination', pp. I025- I034. Roma: Gangemi Editor International Publishing.

Cook K., Geneveive, H. (2019). Digital Heritage as Collaborative Process: Fostering Partnerships, Engagement and Inclusivity in Museums. In IUScholarWorks Journals, 3, I, pp. 83-99.

Gasparetto F., Baratin L. (2020). La rappresentazione del restauro. Quale ruolo per il disegno documentativo come intervento conservativo. In A. Arena et al. Connettere. Un Disegno per annodare tessere. Atti del Convegno UID 2020, pp. 467-484. Milano: FrancoAngeli.

Gustafsson C. (2019). Conservation 3.0 - Cultural Heritage as a driver for regional growth. In SClentific RESearch and Information Technology Ricerca Scientifica e Tecnologie dell'Informazione, vol. 9, Issue I, pp. 2 I-32.

loannides M., Georgopoulos A., Scherer M. (2005). Standards in cultural heritage: the missing grammar for the digital documentation of the past. In Proceedings International Archives of the Photogrammetry, Remote Sensing and Spatial Information Sciences, XXXVI-5/C34 (2005)

Konsa K. (20 I5). Modern conservation: connecting objects, values, and people. In Baltic Journal of Art History, n. 10, pp. 53-84. <https://doi.org/I0.12697/BJAH.2015.10.03> (consultato il 25 gennaio 202I).

Lykourentzou I., Antoniou A. (2019). Digital innovation for cultural heritage: lessons from the European Year of Cultural Heritage. In SClentific RESearch and Information Technology Ricerca Scientifica e Tecnologie dell'Informazione, vol. 9, issue I, pp. 9 I-98.

Munster S. (2017). A Survey on topics, Researchers and Cultures in the field of digital heritage. In ISPRS Annals of the Photogrammetry, Remote Sensing and Spatial Information Sciences (volume IV-2M2), pp. 157-162. Proceedings of 26th International CIPA Symposium 2017 Ottawa, 28 august - 0 I september

Santana Quintero M. et al. (2019). Ethical Framework for Heritage Recording Specialists Apply-ing Digital Workflows for Conservation. In ISPRS The International Archives of the Photogrammetry, Remote Sensing and Spatial Information Sciences (XLII-2 WI5), pp. 1063-070.

\section{Sitografia}

Convenzione di Faro. Convenzione quadro del Consiglio d'Europa sul valore dell'eredità culturale per la società (2005): <https:// www.coe.int/it/web/conventions/full-list/-/conventions/treaty/l99> (consultato il 25 gennaio 2021).

La carta di Londra. Per la visualizzazione digitale dei beni culturali (2009): <www.londoncharter.org> (consultato il 24 gennaio 2021).

Autori

Francesca Gasparetto, Università degli Studi di Urbino, francesca.gasparetto@uniurb.it Laura Baratin, Università degli Studi di Urbino, laura.baratin@uniurb.it

Per citare questo capitolo: Gasparetto Francesca, Baratin Laura (2021). Open Conservation: tecniche di rappresentazione a supporto dell'iter conservativo/Open Conservation: representation techniques to support the conservative process. In Arena A., Arena M., Mediati D., Raffa P. (a cura di). Connettere. Un disegno per annodare e tessere. Linguaggi Distanze Tecnologie. Atti del $42^{\circ}$ Convegno Internazionale dei Docenti delle Discipline della Rappresentazione/Connecting. Drawing for weaving relationship. Languages Distances Technologies. Proceedings of the $42^{\text {th }}$ International Conference of Representation Disciplines Teachers. Milano: FrancoAngeli, pp. 749-764. 


\title{
Open Conservation: Representation Techniques to Support the Conservative Process
}

\author{
Francesca Gasparetto \\ Laura Baratin
}

Abstract

The cultural heritage sector has recently been touched by a digitisation process that is evolving and radically changing the meaning of fruition and valorisation. Although technologies in this field are constantly growing, it is still difficult to define their role in a 'digital conservation' perspective.

This contribution proposes a new approach to the design of conservation practices starting from the use of digital data produced in the context of a restoration laboratory and their different representations.

Knowing that the technical processes of digitisation are not enough to obtain a correct conservation, it is necessary to deepen a research able to propose a methodological, organisational, and procedural approach for the evolution of the new conservation. The work proposes a flow organised into three narrative levels. Each of them employs specific approaches in order to make accessible the digital documentation produced within open restoration sites.

The general goal is to define a new theoretical direction, able to include in the conservation process the use of recent technologies in a conscious way and to lay the foundations for the development of the lineaments of an inter- and multidisciplinary theory that guides the future of conservation practices according to a digital logic.

Keywords

conservation, accessibility, participation, digital methodology, narrative levels.

Tthe diagram of documentation flow in a restoration laboratory for planning and representing the conservation practices.
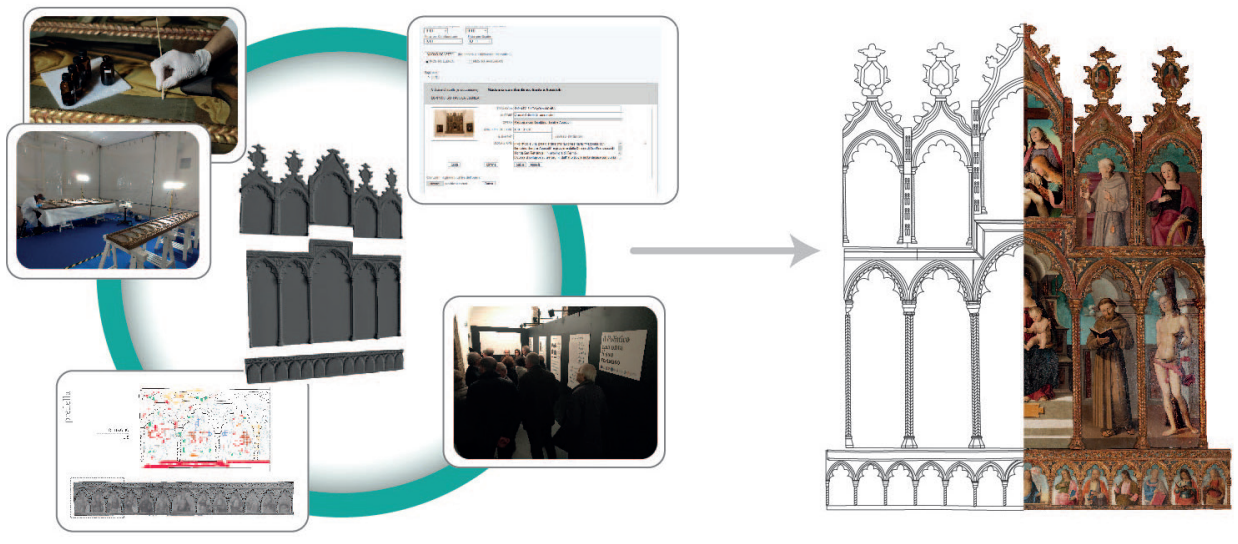


\section{Introduction}

The role of digital documentation and representation for dissemination goals is universally acknowledged in the field of cultural heritage, especially in relation with fruition and valorisation. Nowadays, the digital applications have spread in museum and they have encouraged the use of ICT to access to cultural heritage. The state of the art about dissemination is totally different for the conservation issue, today still addressed to technicians and professionals.

The study would demonstrate the need to deepen the critical area of digital conservation, often forgotten in discussions about digital application for artistic objects. The conservator today is trained in the main technologies and techniques of documentation and his education and digital skills empowers him to a new professional role. In fact, the digital conservation process considers the use of contemporary tools as an opportunity to innovate theory, practice, and general objectives in order to make heritage much more accessible.
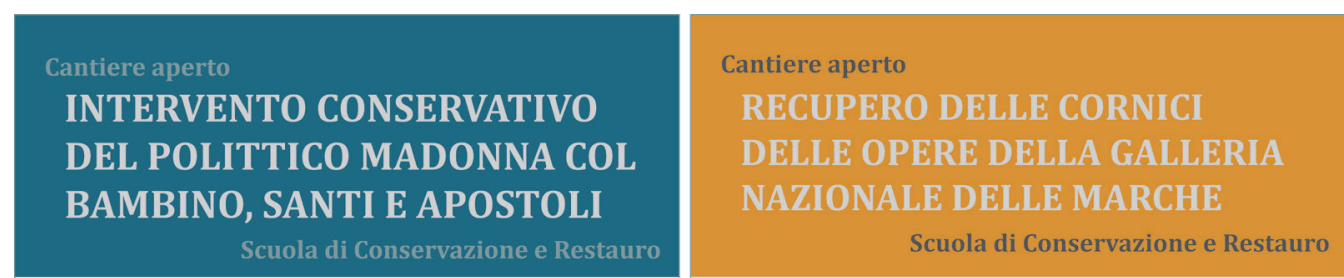

CONVENZIONE TRA ANCI MARCHE | PIO SODALIZIO DEI PICENI | UNIVERSITA' DI URBINO 4 ottobre 2018 delibera n. 140/2018 Convenzione per indagini al fine del
restauro di opere d'arte conservate nel Comuni marchigiani lesionate dal

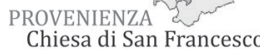
Monte San Pietrangeli (FM)
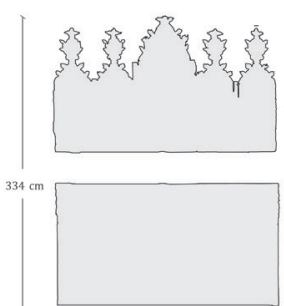

$$
\begin{array}{r}
\text { parti } \\
\text { assemblate }
\end{array}
$$

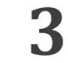

personaggi raffigurati raffigurat

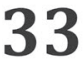

\section{fasi
di lavoro}

ANALISI STORICO-ARTISTICHE 1

RILIEVI GEOMETRICI 2D e 3D 2

INDAGINI DIAGNOSTICHE $\mathbf{3}$

PROGETTO E INTERVENTO 4

COMUNICAZIONE E FRUIZIONE 5

\section{$30 \mathrm{MQ}$ \\ di allestimento del cantiere nell'abside della Pinacoteca di} San Domenico

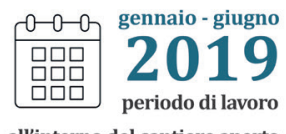

all'interno del cantiere aperto

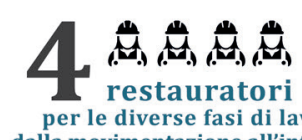

dalla movimentazione all'intervento

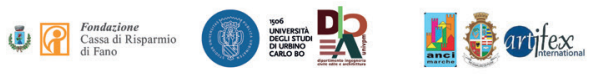

Cantiere aperto

RECUPERO DELLE CORNICI

DELLE OPERE DELLA GALLERIA

Scuola di Conservazione e Restauro

Schedatura digitale e

Mappatura dello stato conservativo delle cornici o parti di cornici

conservate nei depositi di Palazzo Ducale

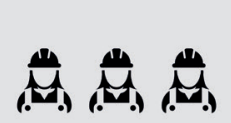

3 restauratori

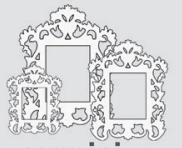

cornici o parti di cornici

La schedatura prevede l'acquisizione di informazioni relative a:

$>$ caratteristiche storico-artistiche

$>$ rilievi dimensionali

$>$ materiali costitutivi

$>$ stato conservativo

questo porta alla redazione di schede conservative dedicate utilizzando i sistemi digitali di documentazione dell'Università di Urbino.

Larchivio digitale fornisce alla Direzione della Galleria Nazionale delle Marche uno strumento per la programmazione degli interventi, al fine del riutilizzo delle cornici nell'allestimento museale $\mathrm{e}$ una ricerca della loro collocazione originale,
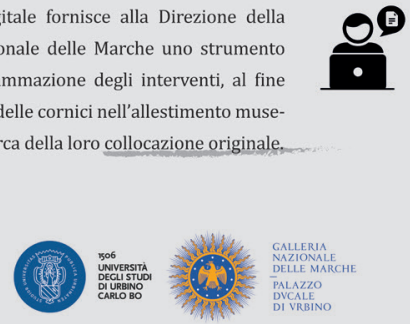


\section{Digital preservation and the european process for its recognition}

In recent years, particularly following 2018 [I], the commitment of European institutions to recognise a specific cultural space for the digital production coming from Heritage studies and surveys has become increasingly concrete. In particular, the European Commission has expressed itself through the approval of charters and documents dedicated to the topics of accessibility and valorisation through digital. The Declaration of Cooperation on Advancing Digitisation signed in 2019 by 27 member countries [2] is a concrete example. In the document, signatory countries commit to supporting cultural policies aimed at digitising monuments and heritage sites and using digital resources to promote citizen involvement in innovative preservation and protection activities. Funding to support important research projects demonstrates the interest. The $4 \mathrm{CH}$ project [3] is the most recent reference. The project is funded thanks H2020 programme and it started in January 202I. It's working on the definition of a Competence Centre for the preservation of Cultural Heritage through fully digital applications.

Recently, researchers engaged in the field of documentation and digitisation have been declaring the need to define new roles of expertise in order to best build the new field of digital [Munster 2017]. Operating ethics [Santana Quintero 2019], identification of the right transmission channels and, most importantly, standards to refer to [loannides et al. 2005; Gasparetto, Baratin 2020] are needed in order to work all in the same direction. It is evident that innovation for Heritage introduces the use of contemporary tools to improve safeguarding practice. In addition to this, it is important to identify what theoretical changes the use of new technologies may bring about. Gustafsson proposes the new concept of Preservation 3.0 [Gustafsson 2019] and takes into account the changing global scenario and the evolution of social culture in order to propose a more active preservation of cultural objects.

The actors in this new process work on several levels because conservation does not only respond to the needs of the goods, but also to those of the citizens, and thus enter a trading zone between practical needs and theoretical needs to be respected. In this sense, those who talk about conservation today must relate the new tools to the needs of an increasingly evolved and responsive information society, designing a participatory approach that preserves buildings and works of art as well as preserving the cultural relationships between objects and citizens.

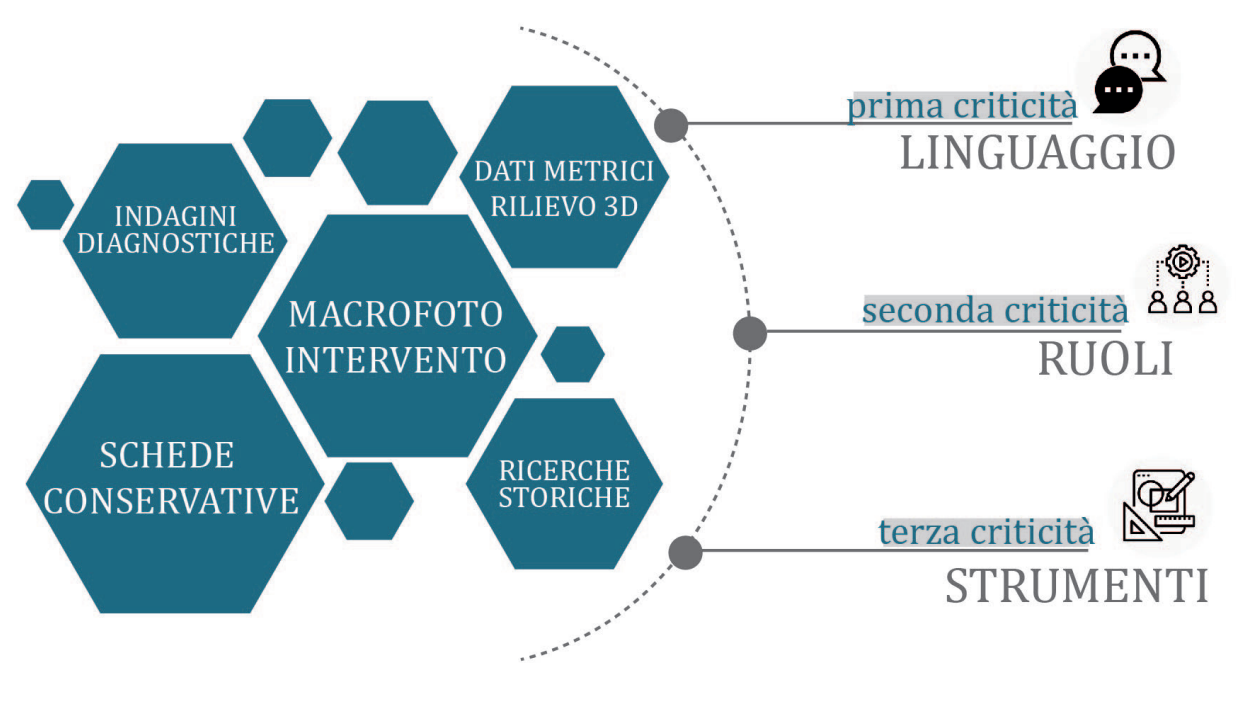




\section{A proposal of methodology}

The School of Conservation and Restoration of the University of Urbino has always explored in depth the role of the 'digital restorer' in the contemporary scenario, both for research and educational fields. Students follow two courses related to Drawing and Digital Representation techniques and, moreover, further lectures about 3D data processing for conservation in the context of practical laboratories. The educational curriculum provides for the transfer of technical-documentary skills in order to supply information about how representing and communicating the intervention. In the same way, the third mission activities are often aimed at involving citizens and visitors in specific exhibitions, designed on research around the theme of digital conservation.

The most emblematic case study conducted in the last period is the setting up of two open laboratories: one for the restoration of the Monte San Pietrangeli Polyptych at the Pinacoteca di San Domenico in Fano and the other for the analysis and documentation of the ancient frames storaged in the depository of the Palazzo Ducale in Urbino (fig. I).

The purpose of both projects was to establish a pro-active conservation scenario, from the documentation of the work carried out on laboratory to the dissemination of information to stimulate the participation of visitors and citizens.

A first part of the experimentation focused on improvement of documentation workflow, in order to relate the digital technologies and for developing a standard model oriented to dynamic data collection [Baratin, Gasparetto 2019; BaratinGasparetto 2020].

The second phase of the study addressed t, he search for a communication method to be applied after the interventions, i.e. after the closing of the worksites.

The research was carried out in different contexts. The study on the artistic frames of Ducal Palace was a short experience. The worksite - which had been set up in the underground spaces of historical building - was stopped due to the Covid-19 emergency. Consequently, it was not possible to further develop the communication strategies launched. About the project that involved the Polyptych, it was possible develop different strategies: firstly, during the open laboratory set up in Fano in 2019; then in the different temporal exhibitions in which the object was displayed in Ascoli Piceno, Rome and Senigallia (2020-202 I); finally, in the project of a special location in which the painting will be relocated in Monte San Pietrangeli in mid-2021.The spaces and the needs are totally different, and they led to design opportunities developing hybrid applications between digital and analogue. Starting from the analysis of the critical points (fig. 2) proposed by the scenario, the communicative model was defined.

The lack of a common language was identified as the first critical issue. In fact, a shared and accepted language is necessary to communicate the collected data correctly. Usually, the technical and scientific data processed during the intervention are systematised within a tailor-made documentation system. However, the digital system is not able to translate the data into information. However, the digital system is not able to translate the data into information. In this case, digital tools provide a fundamental support for the quality of data analysis and management but are not able to elaborate the translation. An open data system was therefore developed, in order to give visitors the possibility to investigate and enter the documentation system, according to specific access authorisations.

The definition of roles was the second critic point. In fact, the Italian legislation on cultural heritage [4] clearly indicates who should be involved in restoration, security, valorisation, and protection. On the other hand, the competences and professional skills for the topic of communication to increase the accessibility of cultural heritage and its preservation have never been clearly defined. The ambiguity of roles leads to a consequent misunderstanding. Restorers are not involved in the application of innovative technological tools and they are not required to develop digital content during the restoration and after the conservation process.

Finally, the third critical issue concerns the approach to data, in particular the choice of technical tools and technologies for their acquisition, analysis, and dissemination. However, there is a lack of shared guidelines and operational protocols to officially validate the use of 
specific technologies for specific purposes. More than ten years ago, the London Charter underlined the importance of standards for digital visualisation, in order to be able to move in a scientifically context. Today it would be necessary to look at general principles for using digital technologies properly in the context of heritage protection and preservation.

Thanks to the efforts made for the Polyptych, a general model was defined, currently being tested. This was designed on the basis of the data collection and the analysis of the general communicative outputs from its restoration. In a early stage of the research, the same model was testing in the context of the documentation of ancient frames of Ducal Palace, in Urbino.

Fig. 3. Proposed scheme of the three levels of communication of data
regarding the conservation process.

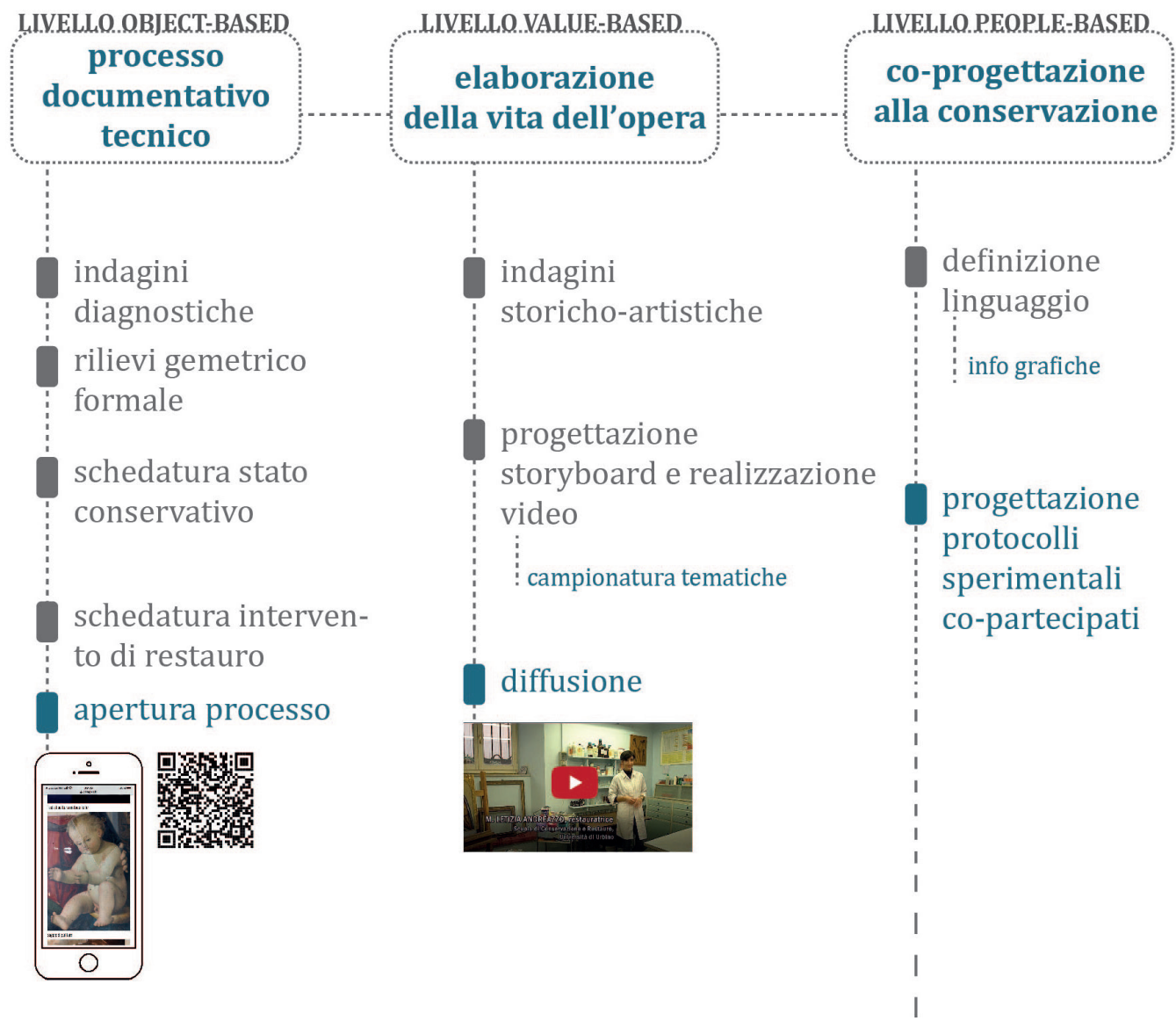

\section{Three levels of narrative for an open conservation}

The experimental proposal was structured into three narrative levels: a technical-scientific level, a historical-artistic level and finally a socio-cultural and identity level. Each level corresponds to different tools for representing the information collected (fig. 3). The documentary flow is therefore organised according to outputs designed specifically for each level. The first level contains more specialised information on the shape, dimensions, materials and restoration work, including descriptive notes on the state of conservation, detailed 
photographs of degradation, etc., as well as technical notes collected during the building work. The persons responsible for this collection have been defined, selected from among restorers and scientific experts. The narrative level was named 'object-based', as the type of representation of the collected data is close to the physical preservation of the object.

The second level defined the 'journey' of the Polyptych and was based on the collection of historical data. The level called 'value-based' emphasises the importance of the art-historical narrative and focuses the value of the object. The two narrative levels above are recognised as passive moments, during which the visitor assimilates information and elaborates it individually.

Finally, the socio-cultural and identity level, called 'people-based', defines an integrated shared ecosystem. Here the main role is not clearly defined. The activity of the community of citizens is integrated with the activity of experts, collaborating in the evolution of the heritage. This step involves the interaction between the different actors, a productive driver enabling an active process.

It is interesting to analyse the development contexts of the three levels. In the case of the open laboratory, the communication was carried out on level one and level two. Here, digital tools provided information about the restoration work in order to allow the participation of non-experts. Thanks to the possibility of framing a QR code, the visitor could explore the records and the images produced by restorers. In this context, the communication is passive, but a digital tool has been identified in order to be able to update the data in real time.

The situation is different for the project of relocating the artwork, which involves together the three narrative levels. In this case, however, the first two levels narrate a completed work. For this reason, the proposed representation use 'fixed' tools, such as graphic panels or video.

For the 'object-based' level, the representation does not require any interaction with public, it is more focused on systematisation of technical data. Starting from the information derived from digital survey, a graphic bidimensional maps was carried out, thanks to the application of representation methods directly adopted from architecture discipline. These methods take advantage of the communicative power of graphic simplification and the semantic reading of colour (fig. 4).

INTERVENTO CONSERVATIVO DEL POLITTICO MADONNA COL BAMBINO, SANTI E APOSTOLI
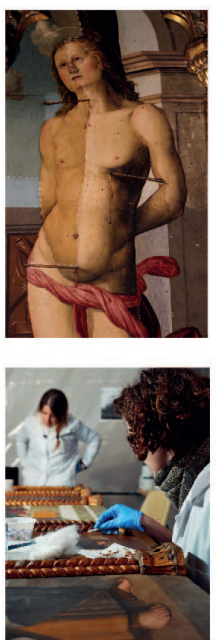
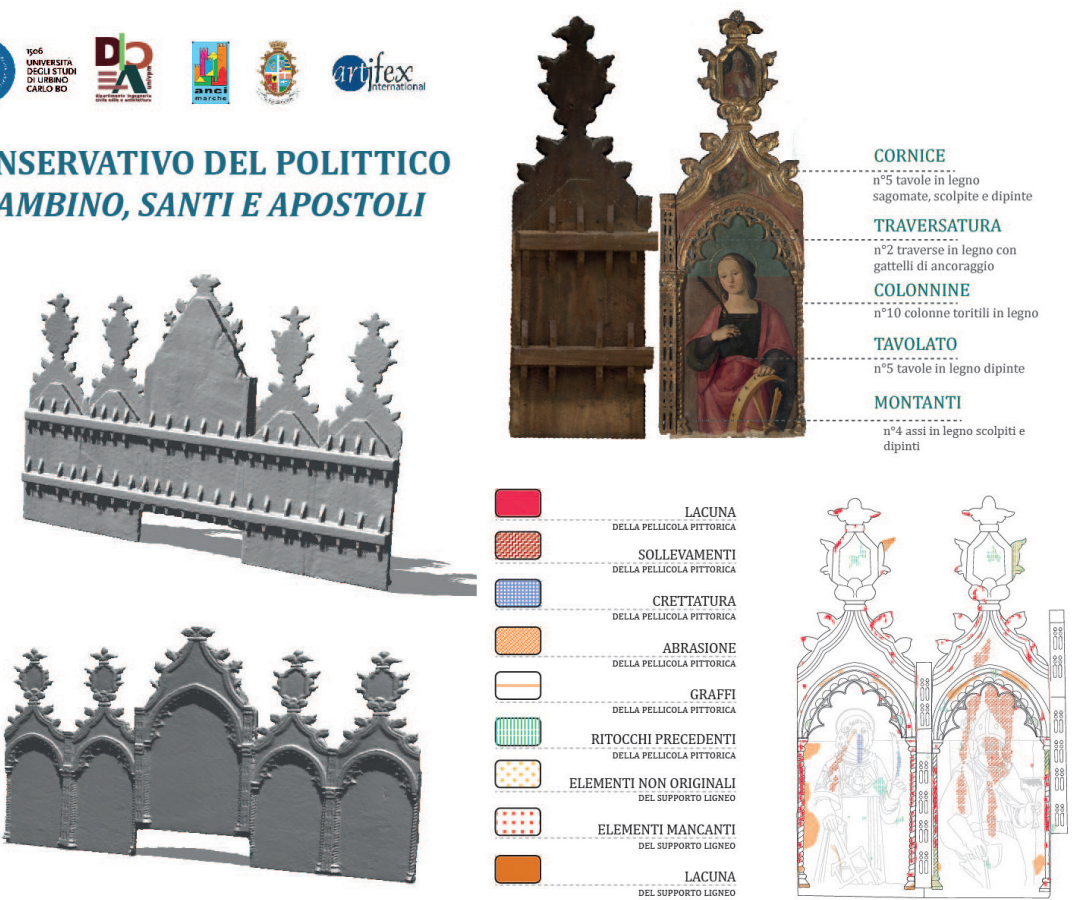
The 'value-based' level was represented by a video. This is the most appropriate tool to convey the idea of evolution and creation of knowledge, using moving images to support evolving data. By sampling the themes, short videos of easy interpretation were produced, involving the actors and the different processes of restoration and valorisation projects [5]. The two narrative levels open up the conservation issue. Starting from this point, it is possible to design co-participatory protocols for conservation and use. The 'people-based' level is introduced in this phase. The work is organised in two steps: a closed project step and an open participatory step.

The first phase defines a graphic language, which will be used in a self-managed way in the participatory phase. In particular, some infographics have been selected to indicate the macro-themes and some specific colours are indicated, already used for the representation of the degradation of the object-based level.

The exhibition space in which the work will be relocated is a multifunctional environment, which will be used by the community of the village of Monte San Pietrangeli for various purposes, in order to be able to enjoy the Polyptych after the important restoration. when the work will arrive around mid-202I, specific activities of valorisation and fruition are planned with a view to re-appropriation of the heritage damaged by the 2016 earthquake.

In this sense, the graphic tools designed will be used at various levels to continue writing the history of the Polyptych. As for the representative methods of the results that will be obtained, these have not yet been defined since the participatory process will lead to the most suitable choice following the proposed method.

\section{Conclusions}

The applied representation methods are aimed at creating an innovative conservation process and not only a conservation product. This kind of approach allows to follow the evolving path to continuous changes and moreover it can adapt to the needs of the heritage and of the community that cares for it.

The proposed research presents a possible translation of a conservative process. The levels are calibrated to the intervention phases and they allow planning an open approach, making technical data accessible and shortening the distance between experts and the community. The skills and tools of the representation disciplines, together with the coordination of the different technologies, had led to the definition of a 'people-centred' information model.

The typically digital approach - which prefers the construction of a participatory network rather than a unidirectional information system - can systematise conservative information and actively transform them.

\section{Notes}

[I] European Year of Cultural Heritage. <https://europa.eu/cultural-heritage> (accessed 202I, January 16).

[2] <https://ec.europa.eu/digital-single-market/en/news/eu-member-states-sign-cooperate-digitising-cultural-heritage> (accessed 2021, January 16).

[3] <https://www.4ch-project.eu> (accessed 2021, January 16).

[4] Reference is made to the Code of Cultural Heritage and Landscape (Legislative Decree 42/2004, art. 30).

[5] There are no pictures of the setting up of the video inside the room because the inauguration of the multifunctional space was postponed due to the Covid-19 emergency. 


\section{References}

Baratin L., Gasparetto F. (2019). Contemporary restoration as an inclusive process. The new digital documentation challenges. Disegnarecon, G. Amoruso, T. Jelenski (a cura di). Experiential Design for Heritage and Environmental Representation, vol. 12 , n. 23, pp. 3.1-3.13.

Baratin L., Gasparetto F. (2020). Digital Cultural Heritage: some notes from conservative experience. In C. Gambardella (ed.). World Heritage and Contamination. Abstracts of the XVIII International Forum 'World Heritage and Contamination', pp. I 025 - I034. Roma: Gangemi Editor International Publishing.

Cook K., Geneveive, H. (2019). Digital Heritage as Collaborative Process: Fostering Partnerships, Engagement and Inclusivity in Museums. In IUScholarWorks Journals, 3, I, pp. 83-99.

Gasparetto F., Baratin L. (2020). La rappresentazione del restauro. Quale ruolo per il disegno documentativo come intervento conservativo. In A. Arena et al. Connettere. Un Disegno per annodare tessere. Atti del Convegno UID 2020, pp. 467-484. Milano: FrancoAngeli.

Gustafsson C. (2019). Conservation 3.0 - Cultural Heritage as a driver for regional growth. In SClentific RESearch and Information Technology Ricerca Scientifica e Tecnologie dell'Informazione, vol. 9, Issue I, pp. 2I-32.

loannides M., Georgopoulos A., Scherer M. (2005). Standards in cultural heritage: the missing grammar for the digital documentation of the past. In Proceedings International Archives of the Photogrammetry, Remote Sensing and Spatial Information Sciences, $\mathrm{XXXVI-5/C34} \mathrm{(2005)}$

Konsa K. (20I5). Modern conservation: connecting objects, values, and people. In Baltic Journal of Art History, n. I0, pp. 53-84. <https://doi.org/1 0.12697/BJAH.2015.10.03> (accessed 202I, January 25).

Lykourentzou I., Antoniou A. (2019). Digital innovation for cultural heritage: lessons from the European Year of Cultural Heritage. In SClentific RESearch and Information Technology Ricerca Scientifica e Tecnologie dell'Informazione, vol. 9, issue I, pp. 9 I-98.

Munster S. (2017). A Survey on topics, Researchers and Cultures in the field of digital heritage. In ISPRS Annals of the Photogrammetry, Remote Sensing and Spatial Information Sciences (volume IV-2M2), pp. 157-162. Proceedings of 26th International CIPA Symposium 2017 Ottawa, 28 august - 0 I september.

Santana Quintero M. et al. (2019). Ethical Framework for Heritage Recording Specialists Apply-ing Digital Workflows for Conservation. In ISPRS The International Archives of the Photogrammetry, Remote Sensing and Spatial Information Sciences (XLII-2/ WI5), pp. 1063-070.

\section{Website}

Convenzione di Faro. Convenzione quadro del Consiglio d'Europa sul valore dell'eredità culturale per la società (2005): <https:// www.coe.int/it/web/conventions/full-list/-/conventions/treaty/l99> (accessed 2021, January 25).

La carta di Londra. Per la visualizzazione digitale dei beni culturali (2009): < www.londoncharter.org> (accessed 202I, January 25).

\section{Authors}

Francesca Gasparetto, Università degli Studi di Urbino, francesca.gasparetto@uniurb.it Laura Baratin, Università degli Studi di Urbino, laura.baratin@uniurb.it

To cite this chapter: Gasparetto Francesca, Baratin Laura (2021). Open Conservation: tecniche di rappresentazione a supporto dell'iter conservativo/Open Conservation: representation techniques to support the conservative process. In Arena A., Arena M., Mediati D., Raffa P. (a cura di). Connettere. Un disegno per annodare e tessere. Linguaggi Distanze Tecnologie. Atti del $42^{\circ}$ Convegno Internazionale dei Docenti delle Discipline della Rappresentazione/Connecting. Drawing for weaving relationship. Languages Distances Technologies. Proceedings of the $42^{\text {th }}$ International Conference of Representation Disciplines Teachers. Milano: FrancoAngeli, pp. 749-764. 